\title{
Pembrolizumab-Induced Type 1 Diabetes in a 95-Year-Old Veteran With Metastatic Melanoma
}

\author{
Damond B. Ng, MD, MPH; Jane Weinreb, MD; and Sara-Megumi Rumrill, MD
}

\section{Low C-peptide levels should prompt a high suspicion for immune checkpoint inhibitor- induced type 1 diabetes, and initiation of insulin therapy should be strongly considered.}

\author{
Author affiliations \\ can be found at the \\ end of the article. \\ Correspondence: \\ Damond $\mathrm{Ng}$ \\ (dng@mednet.ucla.edu)
}

Fed Pract. 2021;38(11). Published online November 12 doi:10.12788/fp.0191
党 mmune checkpoint inhibitors (CPIs) have revolutionized cancer therapy and improved the prognosis for a variety of advanced solid tumors and Hodgkin lymphoma, but evidence is growing regarding severe endocrine disturbances. ${ }^{1,2}$ CPIs block inhibitory molecules on activated $\mathrm{T}$ cells to increase tumor cell destruction but also can breach normal tolerance, resulting in a spectrum of immune-related adverse events (irAE) ${ }^{1,2}$ Programmed cell death-1 (PD1) inhibitors are one type of CPIs. Pembrolizumab is a humanized monoclonal antibody that targets the PD- 1 checkpoint pathway and is approved for the treatment of malignant melanoma and non-small cell lung cancer. ${ }^{3,4}$ When the PD-1 checkpoint pathway is inhibited, T cells targeting cancer are activated, as are autoreactive T cells, such as those regulating pancreatic islet cell survival, which can lead to type 1 diabetes mellitus (T1DM). ${ }^{5}$

\section{CASE PRESENTATION}

A 95-year-old male veteran with long-standing, stable prediabetes was treated with pembrolizumab for stage 4 melanoma. Four months after treatment initiation and 3 weeks after completion of his sixth treatment cycle of pembrolizumab ( $2 \mathrm{mg} / \mathrm{kg}$ every 3 weeks), he presented for surveillance positron emission tomography (PET) and was incidentally found to have a serum glucose of $423 \mathrm{mg} /$ dL. Hypothesis-driven history taking revealed polyuria, polydipsia, and a 12-lb weight loss during the previous 3 months. The patient reported no abdominal pain, nausea, or vomiting. He showed no evidence of pancreatic metastases on recent imaging. His family history was notable for a daughter with T1DM diagnosed at a young age.

On examination, the patient's vital signs were normal aside from a blood pressure of $80 / 40 \mathrm{~mm} \mathrm{Hg}$. His body mass index was
30. He was alert and oriented with comfortable respirations and no Kussmaul breathing. He exhibited dry mucous membranes and poor skin turgor. Laboratory studies revealed $135 \mathrm{mmol} / \mathrm{L}$ sodium (reference, 135-145), $4.6 \mathrm{mmol} / \mathrm{L}$ potassium (reference, 3.6-5.2), $100 \mathrm{mmol} / \mathrm{L}$ chloride (reference, 99-106), bicarbonate of $26.5 \mathrm{mmol} / \mathrm{L}$ (reference, 23-29), serum blood urea nitrogen $27 \mathrm{mg} / \mathrm{dL}$ (reference, $6-24), 1.06 \mathrm{mg} / \mathrm{dL}$ creatinine (reference, $0.74-1.35$ ), and $423 \mathrm{mg} / \mathrm{dL}$ glucose (reference, 70-100), with negative urine ketones. Further studies demonstrated $462 \mu \mathrm{mol} / \mathrm{L}$ fructosamine (reference, 190270), correlating with hemoglobin $A_{1 c}$ $\left(\mathrm{HbA}_{1 \mathrm{c}}\right)$ close to $11.0 \%\left(\mathrm{HbA}_{1 \mathrm{c}}\right.$ was drawn on admission but cancelled by the laboratory for unknown reasons) ${ }^{6,7}$ Later, an inappropriately low C-peptide level of $0.56 \mathrm{ng} / \mathrm{mL}$ (reference, 0.8-3.85) and a negative antiglutamic acid decarboxylase (GAD) antibody titer resulted. The patient was given IV hydration and admitted to the hospital.

With input from endocrinology, the patient was started on 0.3 units per $\mathrm{kg}$ of body weight basal-prandial insulin therapy. Pembrolizumab was held. Six weeks after discharge, his $\mathrm{HbA}_{1 \mathrm{c}}$ was $7.2 \%$, and C-peptide improved to $1.95 \mathrm{ng} / \mathrm{mL}$ and plasma glucose $116 \mathrm{mg} / \mathrm{dL}$. After shared decision making with his health care team, the patient decided against restarting pembrolizumab. The patient reported that his functional status was preserved, and he preferred to take fewer medications at his advanced age. He died comfortably 6 months after this presentation from complications of metastatic melanoma.

\section{DISCUSSION}

Immunotherapy is now an integral part of cancer treatment and can result in endocrine disturbances. ${ }^{1,2}$ Life-threatening irAEs 
are rare and may mimic more common conditions; thus, there is growing recognition of the need to educate health care professionals in appropriate screening and management of these conditions. CPI-induced T1DM is an uncommon but clinically significant event with an incidence of 0.4 to $1.27 \%$ and a median onset of 20 weeks after initiation of therapy (range, 1-228 weeks). ${ }^{8-12}$ In case series from 3 academic centers, 59 to $81 \%$ of patients with CPIinduced T1DM presented with diabetic ketoacidosis (DKA), and only 40 to $71 \%$ of patients were autoantibody positive. ${ }^{13-16}$ These patients are older than those presenting with classic T1DM, often require intensive care unit admission, and nearly invariably require exogenous insulin injections for metabolic control. ${ }^{13-16}$

Based on the later age of onset of cancers that may be treated with CPI, patients with CPI-induced T1DM may be misdiagnosed with T2DM or hyperglycemia from other causes, such as medications or acute illness in the outpatient setting, risking suboptimal treatment.

Given the infrequent incidence and lack of controlled trials, screening and treatment recommendations for CPI-induced T1DM are based on principles derived from case series and expert opinion. Development of polyuria, polydipsia, weight loss, nausea, and/ or vomiting should prompt investigation for possible development or worsening of hyperglycemia, suggestive of development of T1DM. ${ }^{17}$ American Society of Clinical Oncology (ASCO) guidelines recommend that serum glucose be assessed at baseline and with each treatment cycle during induction for 12 weeks, then every 3 to 6 weeks thereafter. ${ }^{17}$ There is no reported association between the number of CPI treatments and the development of DM. ${ }^{8,9}$ Following our patient's fifth pembrolizumab cycle, a random glucose reading was noted to be $186 \mathrm{mg} / \mathrm{dL}$ (Figure 1). Under the ASCO guidelines, ideally the patient would have received close clinical follow-up given the striking increase in plasma glucose compared with prior baseline lower values and perhaps been further evaluated with an anti-GAD antibody titer to screen for TIDM. ${ }^{17}$

This patient's case adds to the published reports of CPI-induced TIDM without DKA and represents the oldest patient experiencing this irAE in the literature. ${ }^{13-16}$ The degree of elevation of his initial fructosamine, which is comparable to an average plasma glucose of approximately $270 \mathrm{mg} / \mathrm{dL}$, belied the rapid rate of rise of his recent plasma glucose. Given the trajectory of glycemic markers and symptoms, one could certainly be concerned about imminent decompensation to DKA. However, fortuitous point-of-care glucose reading prior to surveillance PET resulted in a new critical diagnosis and initiation of treatment.

Assessing the need for inpatient evaluation includes obtaining urine ketones and acidbase status as screening for DKA. ${ }^{17}$ Antibodies and C-peptide can be sent to support diagnosis of new onset TIDM, although the initiation of therapy should not be delayed for these results. ${ }^{17}$ As noted before, many of these patients also are antibody negative. ${ }^{13-16}$ Low Cpeptide levels should prompt a high suspicion for CPI-induced T1DM, and initiation of insulin therapy should be strongly considered. ${ }^{17}$ In a case series of 27 patients, $85 \%$ exhibited a rapid loss of $\beta$-cell function, evidenced by 


\section{FIGURE 2 Letter}

We are starting our mutual patient on a new medication for their malignancy. There can be rare but clinically significant endocrine AEs with this medication that may present first in primary care. We would like you to be aware so we can enhance our shared knowledge, care coordination, and monitoring for any AEs together. Please alert us as soon as possible if you suspect an adverse reaction. We will also continue to follow the patient closely in Oncology.

Cancer type/indication for therapy: metastatic melanoma

Drug name: pembrolizumab

Drug class: Programmed cell death-1 inhibitor, a type of immune checkpoint inhibitor

Endocrine irAE that may present first in primary care and recommendation for initial workup and endocrine consultation:

- Hypothyroidism (8.5\% incidence; $95 \% \mathrm{Cl}, 7.5-9.7 \%)$ : TSH, free thyroxine;

- Hyperthyroidism (3.7\% incidence; 95\% Cl, 2.8-4.7\%): TSH, free thyroxine, TSH receptor antibodies;

- Hypophysitis (1.1\% incidence; 95\% Cl, 0.5-2.6\%): basic metabolic panel, TSH, free thyroxine, morning ACTH and cortisol levels;

- Primary adrenal insufficiency (0.8\% incidence; $95 \% \mathrm{Cl}, 0.3-2.0 \%)$ : basic metabolic panel, morning ACTH and cortisol levels; and

- Diabetes mellitus/hyperglycemia (0.4\% incidence; $95 \% \mathrm{Cl}, 0.2-1.3 \%)$ : finger stick glucose, hemoglobin $\mathrm{A}_{1 \mathrm{c}}$.

Additional irAEs include colitis, hepatitis, myocarditis, nephritis, and pneumonitis and would require further evaluation and management as well. Do not hesitate to contact us if you are concerned for any of these AEs. For more information, we recommend the following 2 review articles:

1. Brahmer JR, Lacchetti C, Schneider BJ, et al; National Comprehensive Cancer Network. Management of immunerelated adverse events in patients treated with immune checkpoint inhibitor therapy: American Society of Clinical Oncology clinical practice guideline. J Clin Oncol. 2018;36(17):1714-1768. doi:10.1200/JCO.2017.77.6385

2. de Filette J, Andreescu C, Cools F, Bravenboer B, Velkeniers B. A systematic review and meta-analysis of endocrinerelated adverse events associated with immune checkpoint inhibitors. Horm Metab Res. 2019;51(03):145-156. doi:10.1055/a-0843-3366

Abbreviations: ACTH, adrenocorticotropic hormone; AE, adverse event; irAE, immune-related AE; TSH, thyroid-stimulating hormone.

Example letter can be used to alert a primary care clinician of a mutual patient started on immune checkpoint inhibitor therapy and can be saved as a template in the electronic health record.

the acute progression to hyperglycemia and low or undetectable levels of C-peptide at diagnosis. ${ }^{9}$ Likewise, our patient had a low Cpeptide level and negative anti-GAD antibody titer but was treated before these results were available. Inpatient admission for close glycemic monitoring may be reasonable; several cases reported prompt diagnosis and avoidance of DKA in this setting. ${ }^{17}$

In contrast to other irAEs, there is no available evidence that high-dose corticosteroids alter the course of pembrolizumabinduced T2DM. ${ }^{18}$ Depending on the degree of hyperglycemia, endocrinology consultation and insulin treatment are appropriate where the diagnosis of TIDM is suspected even without evidence of DKA. ${ }^{17}$ For patients with T2DM, there may be a positive synergistic effect of metformin in combination with CPIs in tumor control. ${ }^{19}$ Our patient's C-peptide improved with insulin treatment, consistent with correction of glucose toxicity and a honeymoon period in his course. However, in patients reported with pembrolizumab-induced T1DM, insulin requirement for treatment generally persists despite cessation of pembrolizumab therapy. ${ }^{13-16}$

\section{CONCLUSIONS}

Pembrolizumab-induced TIDM is a rare, but potentially life-threatening irAE. The acute risk of DKA requires early recognition and prompt treatment of patients taking CPIs. More than $90 \%$ of primary care physicians (PCPs) fulfill general medical care roles for patients with cancer; therefore, they play an essential role in evaluating symptoms during therapy. ${ }^{20}$ Further studies evaluating the role of PCPs and outcomes when PCPs are involved in oncologic care should be conducted.

With increased index of suspicion, this clinical scenario presents an opportunity for PCPs that may help reduce irAE-associated morbidity and mortality of patients on CPIs, like pembrolizumab. Figure 2 illustrates an example addendum that can be used to 
alert and tag a PCP of a mutual patient after initiation of CPI therapy. Determining the optimal interface between PCPs, oncologists, and endocrinologists in delivering and coordinating high-quality cancer care in the setting of immunotherapy is an important area for ongoing quality improvement.

\section{Acknowledgment}

The authors thank all the staff and health care professionals at VA Greater Los Angeles Healthcare System who were involved in the care of this patient.

\section{Author affiliations}

Damond $\mathbf{~ N g}$ is a Resident Physician in the Department of Medicine at David Geffen School of Medicine in Los Angeles, California. Jane Weinreb is Chief of the Division of Endocrinology at the Veterans Affairs (VA) Greater Los Angeles Healthcare System and a Clinical Professor in the Department of Medicine at University of California Los Angeles. SaraMegumi Rumrill is an Assistant Clinical Professor in both the Division of General Internal Medicine at the San Francisco VA Medical Center and the Department of Medicine at the University of California, San Francisco.

\section{Author contributions}

Damond $\mathrm{Ng}$ researched the data and wrote the manuscript. Sara-Megumi Rumrill and Jane Weinreb researched the data and reviewed and edited the manuscript. Damond $\mathrm{Ng}$ is the guarantor of this work.

\section{Author disclosures}

The authors report no actual or potential conflicts of interest with regard to this article.

\section{Disclaimer}

The opinions expressed herein are those of the authors and do not necessarily reflect those of Federal Practitioner, Frontline Medical Communications Inc., the US Government, or any of its agencies. This article may discuss unlabeled or investigational use of certain drugs. Please review the complete prescribing information for specific drugs or drug combinations-including indications, contraindications, warnings, and adverse effects-before administering pharmacologic therapy to patients.

\section{References}

1. Puzanov I, Diab A, Abdallah K, et al; Society for Immunotherapy of Cancer Toxicity Management Working Group. Managing toxicities associated with immune checkpoint inhibitors: consensus recommendations from the Society for Immunotherapy of Cancer (SITC) Toxicity Management Working Group. J Immunother Cancer. 2017;5(1):95. doi:10.1186/s40425-017-0300-z

2. Villa NM, Farahmand A, Du L, et al. Endocrinopathies with use of cancer immunotherapies. Clin Endocrinol (Oxf). 2018;88(2):327-332. doi:10.1111/cen.13483

3. Schachter J, Ribas A, Long GV, et al. Pembrolizumab versus ipilimumab for advanced melanoma: final overall survival results of a multicentre, randomised, open-label phase 3 study (KEYNOTE-006). Lancet. 2017;390(10105):1853-1862. doi:10.1016/S0140-6736(17)31601-X

4. Garon EB, Hellmann MD, Rizvi NA, et al. Five-year overall survival for patients with advanced non-small-cell lung cancer treated with pembrolizumab: results from the phase I KEYNOTE-001 Study. J Clin Oncol. 2019;37(28):2518-
2527. doi:10.1200/JCO.19.00934

5. Ribas A. Tumor immunotherapy directed at PD-1. N Engl J Med. 2012;366(26):2517-2519. doi:10.1056/NEJMe1205943

6. Malmstrom H, Walldius G, Grill V, Jungner I, Gudbjomsdottir S, Hammar N. Frustosamine is a useful indicator of hyperglycemia and glucose control in clinical and epidemiological studies- cross-sectional and longitudinal experience from the AMORIS cohort. PLoS One. 2014;9(10):e111463. doi:10.1371/journal.pone.0111463

7. Skinner S, Diaw M, Mbaye MN, et al. Evaluation of agreement between hemoglobin A1c, fasting glucose, and fructosamine in Senagalese individuals with and without sickle-cell trait. PLoS One. 2019;14(2):e0212552. doi:10.1371/journal.pone.0212552

8. Byun DJ, Wolchok JD, Rosenberg LM, Girotra M. Cancer immunotherapy-immune checkpoint blockade and associated endocrinopathies. Nat Rev Endocrinol. 2017;13(4):195-207. doi:10.1038/nrendo.2016.205

9. Stamatouli AM, Quandt Z, Perdigoto AL, et al. Collateral damage: insulin-dependent diabetes induced with checkpoint inhibitors. Diabetes. 2018;67(8):1471-1480. doi:10.2337/dbi18-0002

10. Liu J, Zhou H, Zhang Y, et al. Reporting of immune checkpoint inhibitor therapy-associated diabetes, 2015-2019. Diabetes Care. 2020;43(7):e79-e80. [Published online ahead of print, 2020 May 11]. doi:10.2337/dc20-0459

11. Barroso-Sousa R, Barry WT, Garrido-Castro AC, et al. Incidence of endocrine dysfunction following the use of different immune checkpoint inhibitor regimens: a systematic review and meta-analysis. JAMA Oncol. 2018;4(2):173182. doi:10.1001/jamaoncol.2017.3064

12. de Filette J, Andreescu CE, Cools F, Bravenboer B, Velkeniers B. A systematic review and meta-analysis of endocrine-related adverse events associated with immune checkpoint inhibitors. Horm Metab Res. 2019;51(3):145156. doi:10.1055/a-0843-3366

13. Hughes J, Vudattu N, Sznol M, et al. Precipitation of autoimmune diabetes with anti-PD-1 immunotherapy. Diabetes Care. 2015;38(4):e55-e57. doi:10.2337/dc14-2349

14. Clotman K, Janssens K, Specenier P, Weets I, De block CEM. Programmed cell death-1 inhibitor-induced type 1 diabetes mellitus. J Clin Endocrinol Metab. 2018;103(9):3144-3154. doi:10.1210/jc.2018-00728

15. Kotwal A, Haddox C, Block M, Kudva YC. Immune checkpoint inhibitors: an emerging cause of insulindependent diabetes. BMJ Open Diabetes Res Care. 2019;7(1):e000591. doi:10.1136/bmjdrc-2018-000591

16. Chang LS, Barroso-Sousa R, Tolaney SM, Hodi FS, Kaiser UB, Min L. Endocrine toxicity of cancer immunotherapy targeting immune checkpoints. Endocr Rev. 2019;40(1):1765. doi:10.1210/er.2018-00006

17. Brahmer JR, Lacchetti C, Schneider BJ, et al; National Comprehensive Cancer Network. Management of immune-related adverse events in patients treated with immune checkpoint inhibitor therapy: American Society of Clinical Oncology Clinical Practice Guideline. J Clin Oncol. 2018;36(17):1714-1768. doi:10.1200/JCO.2017.77.6385

18. Aleksova J, Lau PK, Soldatos G, Mcarthur G. Glucocorticoids did not reverse type 1 diabetes mellitus secondary to pembrolizumab in a patient with metastatic melanoma. BMJ Case Rep. 2016;2016:bcr2016217454. doi:10.1136/bcr-2016-217454

19. Afzal MZ, Mercado RR, Shirai K. Efficacy of metformin in combination with immune checkpoint inhibitors (anti-PD-1/anti-CTLA-4) in metastatic malignant melanoma. J Immunother Cancer. 2018;6(1):64. doi:10.1186/s40425-018-0375-1

20. Klabunde CN, Ambs A, Keating NL, et al. The role of primary care physicians in cancer care. $J$ Gen Intern Med. 2009;24(9):1029-1036. doi:10.1007/s11606-009-1058-x 